\title{
In-patient characteristics in a regional secure unit
}

\author{
J. McKenna
}

\begin{abstract}
A survey of 100 completed Reglonal Secure Unit (RSU) in-potient eplsodes shows that most patients are young, mole, short-stay and sufiering from chronic poychotic ctsorders. Substance abuse is prevalent, and many currently foce serlous charges. A signilicant minority of pationts had previously been admilted to the Unil, especially those admitted directly from the community. Nearty haif of all discharges were directly into community placements, and nearty as many remained under the care of the forensic service. Desplite the sampling frame, it is clear that a fow "longstay' patients contribute disproportionately to overall bed occupancy, so that beds for acute admissions may be blocked. Further, 'parallel care' is operating to a signilicant degree in this service, with the inevilable accumulation of patients who will continue to require future in-patient care. These two foctors in particular suggest that the ablitty of the RSU to fulinl its basic functions may become increasingly compromised, particularty with respect to emergency requirements for medlum secure beds of the district lovel.
\end{abstract}

The Edenfield Centre was opened in 1984 as the old North-West Region's Regional Secure Unit (RSU). The Unit aims to provide a service to mentally disordered adults who require assessment, treatment and rehabilitation in conditions of medium security. There are only a few recently published accounts of the in-patient activity of RSUs, though more reports have reviewed experiences from their immediate predecessors, the 'Interim' secure units (ISUs; Bluglass, 1986). In the intervening period, a crisis has developed in terms of demand for in-patient places, so that across England and Wales RSUs (and prtvate sector medium secure beds) are full nearly all the time, and many have 'waiting lists' (Coid, 1993). Any response to this crisis will have to take account of the clinical features of the in-patients currently managed by forensic units, and with this in mind the writer undertook a survey of completed in-patient episodes at the Edenfield Centre.

\section{The study}

In late 1994, the most recent 33 consecutive discharged in-patients were identified to each of the three established consultant forensic psychiatrists (together with one additional 34th patient from the consultant with the quickest turnover), making 100 completed in-patient episodes in all. Only completed and different admissions were included, so that deaths and escapes $(n=4)$ were excluded, and patients admitted more than once during the sampling period ( $n=7$ ) were included only once.

The case notes of each patient were reviewed, using in particular the detailed admission/discharge summaries and psychiatric reports available. Information was recorded on a form which listed various demographic, forensic and clinical issues.

\section{Findings}

Demographic characteristics and legal status

The mean age of the sample was 31.8 years. Twenty-four patients were aged 24 years or less, and 79 were aged between $20-44$ years. Sixteen were female, and 24 were from an ethnic minority group (mainly of Afro-Caribbean origin).

On admission, ten patients were 'informal', and all of these had previously been admitted to the RSU. Four remained subject to section 41 following an earlier conditional discharge from the RSU and were admitted directly from the community without being 'recalled'. Of the remaining six patients, one was transferred directly from police custody without being charged, and discharged home after one week; one was briefly transferred from a Local Authority hostel before going to a District General Hospital (DGH) after one month; one was a formerly 'restricted' patient who periodically requested 'respite' admissions: and three were transferred in crisis from home (for 3 days, 3 weeks and 5 weeks respectively) before returning to their home. Therefore, nine out of the ten patients were admitted from the community, and nine were discharged back into the community.

Thirty-three patients were subject to 'ctvil' (Part II) Sections (Sections 2 and 3 ) of the Mental Health Act (1983). Fifty-seven were detained under 'criminal' (Part III) Sections: 24 were unsentenced prisoners (Sections 35, 36 and 48) 
Table 1. Length of stay by age and legal status

\begin{tabular}{lll}
\hline Category & $\begin{array}{l}\text { No. of } \\
\text { subjects }\end{array}$ & $\begin{array}{l}\text { Length of } \\
\text { stay (weeks) }\end{array}$ \\
\hline Age or less & 24 & 19.0 \\
24 -44 & 62 & 32.3 \\
$25-44$ & 38.1 \\
45 or more & 14 & 30.1 (mean) \\
All patients & 100 & \\
Status & & 5.6 \\
Informal & 10 & 13.7 \\
Civil orders & 33 & 28.9 \\
Criminal orders & 44 & 94.5 \\
Section 41 & 13 & \\
\hline
\end{tabular}

and 18 transferred during sentence under Section 47. Of the 24 prisoners transferred while on remand, 15 went on to receive a Hospital Order (Section 37). In addition, 14 patients were subject to Section 37 at admission, and one patient was recalled under the provisions of Section 41 . Overall, 60 of the 100 patients had no Home Office restrictions on leave, transfer or discharge - either throughout their stay, or at least after criminal proceedings were dealt with.

\section{Length of stay}

The mean length of stay (LOS) is 30.1 weeks, with a skew towards shorter admissions (median LOS $=15$ weeks). Forty patients were discharged within ten weeks, 60 within 20 weeks, and 70 within 30 months. There was also a significant number of much longer stays. Nineteen stayed for over one year, ten for over 18 months, and four for over two years. LOS varied according to age and legal status (see Table 1).

The 'total bed occupancy' represented by the full sample of 100 patients was 3010 patientweeks. Fifty of the patients accounted for $89 \%$ of this total, and the other 50 just $11 \%$. The longeststaying ten patients accounted for $39.7 \%$ of the total occupancy, while the shortest-staying ten patients were responsible for just $0.4 \%$.

\section{Psychiatric history}

In terms of the recorded primary diagnosis, 86 patients suffered from a psychotic disorder (schizophrenia 55, schizo-affective psychosis 8 , bipolar affective disorder 17, organic psychosis 2 , drug-induced psychosis 4), nine from a personality disorder, and five from other conditions (neurotic or developmental disorders). The 80 patients with chronic or relapsing psychoses had a longer LOS than those with other disorders (mean LOS=32.4 v. 21.0 weeks).
The majority of patients had had previous formal psychiatric contact $(88 / 100)$, which had begun some years before the index admission (mean time since first contact $=8.5$ years). Over three-quarters of the sample (76) had previous inpatient admissions, numbering a mean of 5.7 admissions per patient. Most of these episodes were in a DGH, but over a third of patients (37) had previously been in a RSU, and 18 had spent time in a Special Hospital (SH).

\section{Substance abuse}

For present purposes, substance abuse was recorded when physical dependence was documented, where substance abuse had precipitated or repeatedly exacerbated a psychotic disorder, or where such use had been closely linked to dangerous or violent behaviour. On this basis, drug abuse was recorded in 45 patients and alcohol abuse in 31 patients. There was some overlap between these two groups, so that these figures included 19 patients who abused both drugs and alcohol. Overall, 57 patients abused either drugs, alcohol, or both. The prevalence of substance abuse was the same in patients with functional psychoses and in those with other diagnoses. Compared with the remaining patients, drug abusers tended to be younger (mean age $27.0 v$ v. 35.9 years) and stayed for shorter periods (mean LOS 20.1 v. 38.3 weeks). Patients admitted from penal settings had higher rates of these problems $(31 / 45,69 \%$ v. $26 / 55,47 \%)$, especially those who had previously served terms of imprisonment $(30 / 38,79 \%$ v. $27 / 62,44 \%$, $\left.\chi^{2}=5.15, P<0.025\right)$.

\section{Forensic history}

Two-thirds of the sample (67) had at least one previous criminal conviction, and over a third (38) had served custodial terms. Most of the latter group had also been psychiatric in-patients (26/ $38,68 \%)$.

For the 64 patients where an 'index charge' was applicable (i.e. excluding those on 'civil sections' or admitted informally, including patients who were still 'conditionally discharged'), analysis of the main or most serious charge showed that homicide and serious offences against the person accounted for about a third of the sample (actual or attempted homicide 8 , actual, or grievous bodily harm 14). Other assaults (8), use or possession of weapons (5), kddnap (1), sexual assaults (10) and arson (10) together made up a further half.

\section{Methods of referral}

As shown in Table 2, nearly half of the sample were admitted from the criminal justice system 
Table 2. Discharge route and referral origin

\begin{tabular}{lcclc}
\hline & \multicolumn{3}{l}{ Discharge routes } \\
\cline { 2 - 5 } Refenal source & DGH & CMTY & SH & CJS \\
\hline SH $(n=11)$ & - & 8 & 3 & - \\
SENT $(n=18)$ & 1 & 4 & & 12 \\
REMD $(n=27)$ & 9 & 13 & - & 5 \\
CMTY $(n=18)$ & 2 & 14 & 1 & 1 \\
DGH $(n=26)$ & 20 & 4 & 2 & - \\
Totals & 32 & 43 & 7 & 18
\end{tabular}

SH, Special Hospltal; SENT, sentenced prisoner; REMD, remand prisoner: CMTY, community; CJS, criminal justice system; DGH, District General Hospltal.

(CJS), and over a quarter from DGHs. The mean LOS varies according to the referral origin: community 14.6 weeks, DGH transfer 15.4 weeks, sentenced prisoner 27.8 weeks, remand prisoner 46.3 weeks, and SH, 61.9 weeks. The SH group accounted for the highest bed-occupancy, although the DGH group was more than twice as large.

The difficulties leading to admission varied widely according to the referral route. Nearly all 'community' cases were still being followed up by the RSU from a previous admission (15/18). In all cases, there was significant concern about their mental state or behaviour (see below). Patients transferred from penal settings were nearly all actively psychotic in custody $(41 / 45$, $91 \%)$, with a final diagnosis of functional psychosis - despite high rates of confounding drug abuse. All of the 26 DGH transfers bar one were clearly demonstrating significantly dangerous or harmful behaviours at the time of referral. Significant assaultiveness and/or seriously threatening behaviour were noted in $21(81 \%)$ of the DGH group, and 'drug abuse' or 'absconding' were never the sole management difficulties preceding referral.

\section{Routes of discharge}

As shown in Table 2, three-quarters of the sample were either discharged directly to the community or transferred to DGH in-patient units. Most of the remainder were returned to the penal system. especially those who were already sentenced at the time of admission. Overall, no less than 41 of the 100 patients were followed up exclusively by the Edenfield Centre teams after discharge, and all but two of this group were discharged directly to residential placements in the community.

\section{Comments}

Preliminary inspection of in-patient stay figures before conducting the study had revealed that there were important differences between the individual teams in terms of mean length of stay, and in the routes of admission and discharge. The present composite cohort was therefore chosen to try and reduce the risk of individual teams being over- or under-represented, so that any conclusions may instead reflect the interface of the RSU as a whole with other services and the community.

\section{Length of stay}

A sample composed of 'completed' in-patient episodes will of course show a bias towards patients who had relatively short, 'acute' admissions, rather than the 'long-stay' patients. Nevertheless, the 'chronic' patients were significantly present: ten patients out of the 100 accounted for two-fifths of the cohort's total bed occupancy. The age/length of stay figures quoted for the inpatient group also suggest that there was a significant number of more refractory admissions who remained in hospital, while a larger, shortstay client group passed quickly in and out of beds which were not yet 'blocked' (Kennedy et al, 1995). The ability of the RSU to respond quickly, effectively or flexibly to acute difficulties in the services referring potential admissions must be in turn compromised.

The Edenfield Centre's figures for length of stay are broadly similar to those reported from other RSUs and ISUs, where mean stays are quoted as being in the range of 7-9 months. The present sample also resembles these reports in being predominantly male and young, and in having a significant proportion of patients from ethnic minorities (see Grounds et al, 1993).

\section{Psychiatric history}

Regarding diagnosis, the sample was heavily weighted towards the functional psychoses, especially those classified as schizophrenic or schizoaffective (who accounted for nearly two-thirds of the sample), while patients with a recorded primary diagnosis of personality disorder (including psychopathic disorder) were far less common $(9 \%)$. These figures again correspond to reports from other medium secure settings.

\section{Substance abuse}

In such a group, the finding of problematic current substance (drug and alcohol) abuse in over half of the sample (irrespective of diagnostic group) is of particular concern. In particular, drug abuse was documented in nearly half of the sample $(n=45)$. The prevalence of substance abuse was rather higher in those admitted from the penal system. and was significantly more common in those with a previous history of imprisonment. The potential 
impact of such a problem in an in-patient unit, in terms of management, prognosis and treatment, may easily be underestimated (see Smith \& Hucker, 1994).

\section{Methods of referral and routes of discharge}

Accounts of the functioning of RSUs may emphasise a particular 'service triangle' in which they operate, marked out by district psychiatric services, the penal system and the SHs. The findings that about one-fifth of these patients were admitted directly from the community, and that over twice this proportion was discharged along the same route, would rather suggest a 'service square'. Community admissions - who also accounted for nearly all of the 'informal' admissions - were not prompted by minor or non-clinical problems. There were 18 patients in this group, and nine were readmitted because of a suspected deterioration in their mental state (positive psychotic symptoms, suicidal ideation or behaviour), seven because of dangerous or potentially criminal behaviour in the community, and two because of potentially serious 'breaches' of the terms of conditional discharges. Of the whole group, 15 had previously been admitted to the RSU (including 4 who had also been in an SH), were being followed up by the forensic service at the time of the current admission, and remained under its supervision after discharge; 17 had a criminal record, and one faced serious charges; ten posed no significant management problems during their admission; 14 were discharged back into the community, three to a DGH, and one to an SH.

It is clear that service planning must allow for the provision of beds which would be available for relatively short stays, and at short notice. Whether such beds always need to be 'secure' is less clear - particularly as the 'community' patients stayed only briefly (mean LOS $=14.6$ weeks, and for 'informal' patients, mean LOS $=5.6$ weeks), often remained 'informal' and presented no management difficulties during their stay, and usually were discharged directly back into the community. However, there seems to be some grounds for maintaining care within a forensic service: many have noteworthy 'forensic' pedigrees, have failed to be contained in DGHs previously, are well-known to the forensic service, and an important group remains subject to conditional discharge.

Further, seriously mentally disordered individuals - especially those with violent and dangerous histories - are a group for whom adequate and comprehensive community placements and supports are especially important. Where such facilities are not available, the likely effects will include a 'silting up' at the medium security level, and frequent, urgent re-admissions directly from residential settings.

There are grounds to believe that the proportion of patients who are admitted directly from the community has been increasing in recent years. Higgo \& Shetty (1991) found that $24 \%$ of admissions to the Mersey Region's RSU were of this type, and that more recently there had been an increase in the proportion who were 'readmissions' to the RSU - the community being the primary source of re-admissions. At the Edenfield Centre, 15 of the 18 community admissions were readmissions. Overall, 35 of the 100 patients had been previously admitted to the RSU, and 41 were subsequently 'followed up' by the Unit. Therefore, a substantial proportion of the RSU's in-patient throughput involves a model of service provision that is 'parallel', rather than 'integrated' (Gunn, 1976). Since this group is both relatively young and suffers from serious morbidity (which has already required significant service use over several years), those patients discharged to the community and remaining 'on the books' will potentially require further admissions. The number of such people within a forensic service must presumably continue to increase over time, as individuals accumulate in a 'parallel' service.

It is not clear how the pressure of this gathering group might be offset or accommodated, and how other agencies or services might be part of a response. In the meantime, the Edenfield Centre - like other similar units - is already almost permanently full, and continues to be subject to substantial and critical pressure from the criminal-justice system, the SHs, and the district-level general and specialist services. As well as creating difficulties for patients waiting to be admitted from other secure settings (SHs, prisons), there is a particular problem in the ability of the service to respond to acute problems or crises in settings of lower security (especially the DGHs).

Increased attention may suitably be given to those patients whose clinical characteristics and needs extend well beyond those represented by the 'prototypical', young, acutely ill and shortstay RSU in-patient. It seems clear that many patients do not present serious management problems or challenging behaviours for much of their stay - significant numbers enjoy 'leave' privileges beyond the Unit perimeter, and many are ultimately discharged directly to non-secure or residential settings. Acute containment and management in secure conditions as part of wider, integrated psychiatric service is a fundamental role of RSUs, but it may be that forensic clinicians must equally consider novel approaches to the issues of long-term supervision, rehabilitation and resettlement of those suffering from refractory and enduring disorders. 


\section{References}

Buglass, R. (1986) The development of Regional Secure Units. In Secure Provision (ed. L. Gostin). London: Tavistock.

Com, J. W. (1993) A Survey of Bed Occupancy in Medium Securty. 21 st November, 1993. Discussion Paper. Department of Health.

GUNN, J. (1976) Management of the mentally abnormal offender: integrated or parallel. Proceedings of the Royal Society of Medicine, 70, 877-880.
Higco, R. \& SHETTY, G. (1991) Four years' experience of a Reglonal Secure Unit. Joumal of Forensic Psychiatry. 2. 202-209.

SMITH, J. \& HUCKER, S. (1994) Schizophrenia and substance abuse. British Joumal of Psychiatry. 166, 13-21.

J. McKenna, Senior Registrar in Forensic Psychiatry, Edenfield Centre, Mental Health Services, Bury New Road, Prestwich, Manchester M25 3BL

\section{Management for Psychiatrists}

\section{Second Edition}

\section{Edited by Dinesh Bhugra and Alistair Burns}

Since the last edition rapid changes in the NHS have meant that clinicians have had even less time to manage change and keep up to date with health reforms. For this new edition, all the existing material has been extensively revised. In addition, eight new chapters have been added, including a section on changes and conflicts covering large areas of potential difficulty that clinicians may have to deal with.

As before, the emphasis is on how to get the best for and from services. Practical advice is given on management. Negotiation techniques and time and stress management are also covered.

- $220.00 \bullet 360 p p . \odot 1995 \bullet$ ISBN 0902241850

Available from bookshops and from the Publications Department, Royal College of Psychiatrists, 17 Belgrave Square, London SW1X 8PG (Tel. 0171-235 2351 extension 146) 